\title{
Fixed Point Results by Altering Distances in Fuzzy Metric Spaces
}

\author{
Ibtisam Masmali1, Sumitra Dalal1, Nasir Rehman² \\ ${ }^{1}$ College of Science, Jazan University, Jazan, kingdom of Saudi Arabia \\ ${ }^{2}$ Allama Iqbal Open University, Islamabad, Pakistan \\ Email: ibtisam234@hotmail.com, mathsqueen d@yahoo.com, nasirzainy1@hotmail.com
}

Received 17 April 2015; accepted 25 May 2015; published 28 May 2015

Copyright $\odot 2015$ by authors and Scientific Research Publishing Inc.

This work is licensed under the Creative Commons Attribution International License (CC BY).

http://creativecommons.org/licenses/by/4.0/

(c) (1) Open Access

\begin{abstract}
We establish fixed point theorems in complete fuzzy metric space by using notion of altering distance, initiated by Khan et al. [Bull. Austral. Math. Soc. 30 (1984), 1-9]. Also, we find an affirmative answer in fuzzy metric space to the problem of Sastry [TamkangJ. Math., 31(3) (2000), 243-250].
\end{abstract}

\section{Keywords}

Fixed Points, Fuzzy Metric Spaces, Altering Distance, Fixed Point Results

\section{Introduction}

The concept of fuzzy sets was introduced by Zadeh. With the concept of fuzzy sets, the fuzzy metric space was introduced by Kramosil and Michalek [1]. Grabiec [2] proved the contraction principle in the setting of fuzzy metric space. Also, George and Veermani [3] modified the notion of fuzzy metric space with the help of continuous t-norm. Fuzzy set theory has applications in applied sciences such as neural network theory, stability theory, mathematical programming, modelling theory, engineering sciences, medical sciences (medical genetics, nervous system), image processing, control theory and communication.

Boyd and Wong [4] introduced the notion of $\Phi$-contractions. In 1997, Alber and Guerre-Delabriere [5] defined the $\phi$-weak contraction which was a generalization of $\Phi$-contractions. Many researchers studied the notion of weak contractions on different settings which generalized the Banach Contraction Mapping Principle. Another interesting and significant fixed point results as a generalization of Banach Contraction Principle have been established by using the notion of alerting distance function, a new notion propounded by Khan et al. [6]. Altering Distance Functions are control functions which alter the distance between two points in a metric space. For more details, we refer to [6]-[12].

Sastry et al. [13] proved the following: 
Theorem 2.4 [13] Let $(A, S)$ and $(B, T)$ be weakly commuting pairs of self mappings of a complete metric space $(X, \mathrm{~d})$ satisfying

1) $\mathrm{A}(X) \subset \mathrm{T}(X), \mathrm{B}(X) \subset S(X)$

2) There exists $h \in[0,1)$ such that $\psi(d(A x, B y)) \leq h M_{\psi}(x, y)$, where

$$
\begin{aligned}
& M_{\psi}(x, y)=\max \left\{\psi(d(S x, T y)), \psi(d(A x, S x,)), \psi(d(B y, T y)), \frac{1}{2}[\psi(d(A x, T y))+\psi(d(B y, S x))]\right\}, \\
& \forall x, y \in X .
\end{aligned}
$$

and $\psi: \mathrm{R}^{+} \rightarrow \mathrm{R}^{+}$is continuous at zero, monotonically increasing, $\psi(2 t) \leq 2 \psi(t)$ and $\psi(t)=0$ if and only if $t=0$. Suppose that $A$ and $S$ are $\psi$-compatible and $S$ is continuous. Then $A, B, S$ and $T$ have a unique common fixed point.

On the basis of theorem 2.4 of [13], Sastry posed the following open problem:

Is theorem 2.4 of [13] valid if we replace continuity of $S$ by continuity of $A$ ?

In this paper, we prove common fixed point theorems which provide an affirmative answer to the above question on existence of fixed point in fuzzy metric spaces.

\section{Preliminaries}

To set up our results in the next section, we recall some basic definitions.

Definition 2.1 [14] A fuzzy set $A$ in $X$ is a function with domain $X$ and values in $[0,1]$.

Definition 2.2 [14] A binary operation *: $[0,1] \times[0,1] \rightarrow[0,1]$ is a continuous t-norm if $([0,1], *)$ is a topological abelianmonoid with unit 1 such that. $a \times b \leq c \times d$ whenever $a \leq c, b \leq d, \forall a, b, c, d \in[0,1]$

Definition 2.3 [15] The 3-tuple $(X, M, *)$ is called a fuzzy metric space if $X$ is an arbitrary set, * is a continuous $t$-norm and $M$ is a fuzzy set on $X^{2} \times[0, \infty)$ satisfying the following conditions:

$(\mathrm{FM}-1) M(x, y, t)>0$ and $M(x, y, 0)=0$,

(FM-2) $M(x, y, t)=1$ if $x=y$,

(FM-3) $M(x, y, t)=M(y, x, t)$,

$(\mathrm{FM}-4) M(x, y, t) * M(y, z, s) \leq M(x, z, t+s)$,

(FM-5) $M(x, y, t):(0, \infty) \rightarrow[0,1]$ is continuous, for all $x, y, z \in X$ and $s, t>0$.

We note that $M(x, y, \cdot)$ is non-decreasing for all $x, y \in X$.

Definition 2.4 Let $(X, M, *)$ be a fuzzy metric space. A sequence $\left\{x_{n}\right\}$ is said to be

1) G-Cauchy (i.e., Cauchy sequence in sense of Grabiec [5]) if $\lim _{n \rightarrow \infty} M\left(x_{n+p}, x_{n}, t\right)=1$ for all $t>0$ and each $p>0$.

2) Convergent to a point $x \in X$ if $\lim _{n \rightarrow \infty} M\left(x_{n}, x, t\right)=1$ for all $t>0$.

Definition 2.5 [16]-[18] A pair of self mappings $(f, g)$ on fuzzy metric space $(X, M, *)$ is said to be reciprocally continuous if

$$
\lim _{n \rightarrow \infty} f g x_{n}=f z, \lim _{n \rightarrow \infty} g f x_{n}=g z
$$

whenever $\left\{x_{n}\right\}$ is a sequence in $X$ such that

$$
\lim _{n \rightarrow \infty} f\left(x_{n}\right)=\lim _{n \rightarrow \infty} g\left(x_{n}\right)=z
$$

for some $z$ in $X$.

Definition 2.6 An altering distance function or control function is a function $\psi:[0, \infty) \rightarrow[0, \infty)$

such that the following axioms hold:

1) $\psi$ is monotonic increasing and continuous;

2) $\psi(t)=0$ if and only if $t=0$.

Lemma 2.1 [5]. Let $(X, M, *)$ be fuzzy metric space and for all $x, y \in X, t>0$ and if for a number $k \in(0,1), M(x, y, k t) \geq M(x, y, t)$. Then $x=y$.

Lemma 2.2 [5]. Let $\left(X, M,{ }^{*}\right)$ be fuzzy metric space and $\left\{y_{n}\right\}$ be a sequence in $X$. If there exists a number $k \in(0,1)$ such that $M\left(y_{n}, y_{n+1}, k t\right) \geq M\left(y_{n-1}, y_{n}, t\right)$ for all $t>0$ and $n=1,2, \cdots$

Then $\left\{y_{n}\right\}$ is a Cauchy sequence in $X$.

Lemma 2.3 Let $\psi:[0,1] \rightarrow[0,1]$ is continuous and decreasing $\psi(t)=1$ if and only if $t=1$. Then $\left\{t_{n}\right\} \subset[0,1]$ and $\psi\left(t_{n}\right) \rightarrow 1$ implies $t_{n} \rightarrow 1$. 
Definition 2.7 [13] A pair of self mappings $(f, g)$ on fuzzy metric space $\left(X, M,{ }^{*}\right)$ is said to be $\psi$-compatible if

$$
\lim _{n \rightarrow \infty} \psi\left(M\left(f g x_{n}, g f x_{n}, t\right)\right)=1
$$

whenever $\left\{x_{n}\right\}$ is a sequence in $X$ such that

$$
\lim _{n \rightarrow \infty} f\left(x_{n}\right)=\lim _{n \rightarrow \infty} g\left(x_{n}\right)=z
$$

for some $z$ in $X$.

\section{Main Results}

Theorem 3.1 Let $(A, S)$ and $(B, T)$ be weakly commuting pairs of self mappings of a complete fuzzy metric space $(X, M, *)$ satisfying

(3.1) $A(X) \subset T(X), \mathrm{B}(X) \subset S(X)$

(3.2) There exists $h \in[0,1)$ such that $\psi(M(A x, B y, h t)) \leq h M_{\psi}(x, y)$, where

$$
\begin{aligned}
M_{\psi}(x, y)= & \{\psi(M(S x, T y, t)) \times \psi(M(A x, S x, t)) \times \psi(M(B y, T y, t)) \\
& \times \psi(M(A x, T y, \alpha t)) \times \psi(M(B y, S x,(2-\alpha) t))\}
\end{aligned}
$$

$\forall x, y \in X, \alpha \in(0,2), \mathrm{a} \times \mathrm{b}=\min \{a, b\}$. Suppose that $A$ and $S$ are $\psi$-compatible and $A$ is continuous. Then $A$, $B, S$ and $T$ have a unique common fixed point.

Proof: Let $x_{0}$ be any fixed point in $X$. Define sequences $\left\{x_{n}\right\}$ and $\left\{y_{n}\right\}$ in $X$ given by the rule

(3.3) $y_{2 n}=A x_{2 n}=T x_{2 n+1}$ and $y_{2 n+1}=B x_{2 n+1}=S x_{2 n+2}$

This can be done by virtue of (3.1). Now, we prove that $\left\{y_{n}\right\}$ is a Cauchy sequence. For $\alpha=1-q, q \in(0,1)$ in (3.2), we have

$$
\begin{aligned}
\psi & \left(M\left(y_{2 n}, y_{2 n+1}, h t\right)\right)=\psi\left(M\left(A x_{2 n}, B x_{2 n+1}, h t\right)\right) \\
\leq & M_{\psi}\left(x_{2 n}, x_{2 n+1}, t\right) \\
= & \left\{\psi\left(M\left(S x_{2 n}, T x_{2 n+1}, t\right)\right) \times \psi\left(M\left(A x_{2 n}, S x_{2 n}, t\right)\right) \times \psi\left(M\left(B y_{2 n+1}, T y_{2 n+1}, t\right)\right)\right. \\
& \left.\times \psi\left(M\left(A x_{2 n}, T x_{2 n+1},(1-q) t\right)\right) \times \psi\left(M\left(B x_{2 n+1}, S x_{2 n},(1+q) t\right)\right)\right\} \\
= & \left\{\psi\left(M\left(y_{2 n-1}, y_{2 n}, t\right)\right) \times \psi\left(M\left(y_{2 n}, y_{2 n-1}, t\right)\right) \times \psi\left(M\left(y_{2 n+1}, y_{2 n}, t\right)\right)\right. \\
& \left.\times \psi\left(M\left(y_{2 n}, y_{2 n},(1-q) t\right)\right) \times \psi\left(M\left(y_{2 n+1}, y_{2 n-1},(1+q) t\right)\right)\right\} \\
= & \left\{\psi\left(M\left(y_{2 n-1}, y_{2 n}, t\right)\right) \times \psi\left(M\left(y_{2 n+1}, y_{2 n}, t\right)\right) \times \psi\left(M\left(y_{2 n+1}, y_{2 n-1},(1+q) t\right)\right)\right\} \\
= & \left\{\psi\left(M\left(y_{2 n-1}, y_{2 n}, t\right)\right) \times \psi\left(M\left(y_{2 n+1}, y_{2 n}, t\right)\right)\right\} \\
= & \min \left\{\psi\left(M\left(y_{2 n-1}, y_{2 n}, t\right)\right), \psi\left(M\left(y_{2 n+1}, y_{2 n}, t\right)\right)\right\}
\end{aligned}
$$

As $M\left(y_{2 n+1}, y_{2 n-1},(1+q) t\right) \geq M\left(y_{2 n-1}, y_{2 n}, t\right) \times M\left(y_{2 n}, y_{2 n+1}, q t\right)$

If

$$
\min \left\{\psi\left(M\left(y_{2 n-1}, y_{2 n}, t\right)\right), \psi\left(M\left(y_{2 n+1}, y_{2 n}, t\right)\right)\right\}=\psi\left(M\left(y_{2 n+1}, y_{2 n}, t\right)\right),
$$

a contradiction and hence

$$
\psi\left(M\left(y_{2 n}, y_{2 n+1}, h t\right)\right) \leq \psi\left(M\left(y_{2 n-1}, y_{2 n}, t\right)\right),
$$

but as $\psi$ is decreasing so we have $M\left(y_{2 n}, y_{2 n+1}, h t\right) \geq M\left(y_{2 n-1}, y_{2 n}, t\right)$ and hence by lemma (2.2), the sequence $\left\{y_{n}\right\}$ is a Cauchy sequence in $X$. Since $X$ is complete, there is a point $z$ in $X$ such that

(3.4) $y_{2 n}=A x_{2 n}=T x_{2 n+1} \rightarrow z$ and $y_{2 n+1}=B x_{2 n+1}=S x_{2 n+2} \rightarrow z$ as $n \rightarrow \infty$.

Now, suppose that $A$ and $S$ are $\psi$-compatible then we have

(3.5) $A x_{2 n} \rightarrow z$ and $S x_{2 n} \rightarrow z$ implies that $\lim _{n \rightarrow \infty} \psi\left(M\left(A S x_{2 n}, S A x_{2 n}, t\right)\right)=1$ 
Also, $A$ is continuous, so by (3.3),

(3.6) $A A x_{2 n} \rightarrow z$ and $A S x_{2 n} \rightarrow A z$ as $n \rightarrow \infty$

We claim that $\lim _{n \rightarrow \infty} S A x=A z$. By (3.5), we get

$$
\psi\left(M\left(S A x_{2 n}, A z, t\right)\right) \geq \psi\left(M\left(S A x_{2 n}, A S x_{2 n}, \frac{t}{2}\right)\right) * M\left(A S x_{2 n}, A z, \frac{t}{2}\right) \rightarrow 1
$$

as $n \rightarrow \infty$. By lemma (2.3) $M\left(S A x_{2 n}, A z, t\right) \rightarrow 1$ as $n \rightarrow \infty$ and so $\lim _{n \rightarrow \infty} S A x_{2 n}=A z$.

Also, since $A X \subset T X, A z=T w$ for some $w$ in $X$ and corresponding to each $x_{2 n}$, there exists a $w_{2 n}$ such that $A A x_{2 n}=T w_{2 n}$. Thus, we have $A A x_{2 n}=T w_{2 n}=T w$ and $S A x_{2 n} \rightarrow T w$. Also, since $B X \subset S X$, corresponding to each $w_{2 n}$, there exists a $u_{2 n}$ such that $S u_{2 n}=B w_{2 n}$. Thus we have $S u_{2 n}=B w_{2 n} \rightarrow T w$ and $T w_{2 n} \rightarrow T w$.

Now, we claim that $A u_{2 n} \rightarrow T w$ as $n \rightarrow \infty$. Using (3.2) with $\alpha=1$

$$
\begin{aligned}
\psi & \left(M\left(A u_{2 n}, B w_{2 n}, h t\right)\right) \leq M_{\psi}\left(u_{2 n}, w_{2 n}, t\right) \\
= & \left\{\psi\left(M\left(S u_{2 n}, T w_{2 n}, t\right)\right) \times \psi\left(M\left(A u_{2 n}, S u_{2 n}, t\right)\right) \times \psi\left(M\left(B w_{2 n}, T w_{2 n}, t\right)\right) .\right. \\
& \left.\times \psi\left(M\left(A u_{2 n}, T w_{2 n}, t\right)\right) \times \psi\left(M\left(B w_{2 n}, S u_{2 n}, t\right)\right)\right\}
\end{aligned}
$$

Letting $n \rightarrow \infty$,

$$
\psi\left(M\left(A u_{2 n}, T w, h t\right)\right) \leq \psi\left(M\left(A u_{2 n}, T w, t\right)\right),
$$

as $\psi$ is decreasing, so we have

$$
M\left(A u_{2 n}, T w, h t\right) \geq M\left(A u_{2 n}, T w, t\right) .
$$

Thus, we have $A u_{2 n} \rightarrow T w$ as $n \rightarrow \infty$.

Also, we claim that $B w=T w$. Using (3.2) for $\alpha=1$

$$
\begin{aligned}
\psi & \left(M\left(A u_{2 n}, B w, h t\right)\right) \leq M_{\psi}\left(u_{2 n}, w, t\right) \\
= & \left\{\psi\left(M\left(S u_{2 n}, T w, t\right)\right) \times \psi\left(M\left(A u_{2 n}, S u_{2 n}, t\right)\right) \times \psi(M(B w, T w, t))\right. \\
& \left.\times \psi\left(M\left(A u_{2 n}, T w, t\right)\right) \times \psi\left(M\left(B w, S u_{2 n}, t\right)\right)\right\}
\end{aligned}
$$

Letting $n \rightarrow \infty$, we get $M(T w, B w, h t) \geq M(T w, B w, t)$ Thus, we have $T w=B w$

Again, since $B X \subset S X$, so there exists $u$ in $X$ such that $B w=S u$. That is $T w=B w=S u$. Lastly, we show that $A u=S u$. Then by (3.2) with $\alpha=1$, we have

$$
\begin{aligned}
\psi & (M(A u, S u, h t))=\psi(M(A u, B w, h t)) \leq M_{\psi}(u, w, t) \\
= & \{\psi(M(S u, T w, t)) \times \psi(M(A u, S u, t)) \times \psi(M(B w, T w, t)) \\
& \times \psi(M(A u, T w, t)) \times \psi(M(B w, S u, t))\}
\end{aligned}
$$

(3.7) This gives $A u=S u$ and hence we have $A u=S u=B w=T w$.

As $\mathrm{A}$ and $\mathrm{S}$ are weakly commuting, we have $A S u=S A u$ and hence

(3.8) $A A u=A S u=S A u=S S u$.

Also, $B$ and $T$ are weakly commuting, we get

(3.9) $B B w=B T w=T B w=T T w$.

Finally, we show that $A A u=A u$. Again using (3.5), (3.6) and (3.2) with $\alpha=1$.

$$
\begin{aligned}
& \psi(M(A A u, A u, h t))=\psi(M(A A u, B w, h t)) \\
& \leq M_{\psi}(A u, w, t)=\psi(M(A A u, B w, t))
\end{aligned}
$$

which gives that $A A u=A u$. Therefore $A u$ is a common fixed point of $A$ and $S$. Similarly, we can show that $B B w=B w$ and since $T B w=B B w$, we have $B w$, a common fixed point of $B$ and $T$. Finally, $A u=B w$, we have $A u$ as a common fixed point for $A, B, S$ and $T$. The uniqueness follows from 2) and hence the theorem.

Theorem 3.2 Let $(A, S)$ and $(B, T)$ be weakly commuting pairs of self mappings of a complete fuzzy 
metric space $(X, M, *)$ satisfying

(3.10) $A(X) \subset T(X), \mathrm{B}(X) \subset S(X)$

(3.11) There exists $h \in[0,1)$ such that $\psi(M(A x, B y, h t)) \leq h M_{\psi}(x, y)$, where

$$
\begin{aligned}
M_{\psi}(x, y)= & \{\psi(M(S x, T y, t)) \times \psi(M(A x, S x, t)) \times \psi(M(B y, T y, t)) \\
& \times \psi(M(A x, T y, \alpha t)) \times \psi(M(B y, S x,(2-\alpha) t))\}
\end{aligned}
$$

$\forall x, y \in X, \alpha \in(0,2), \mathrm{a} \times \mathrm{b}=\min \{a, b\}$. Suppose that $A$ and $S$ are $\psi$-compatible pair of reciprocal continuous mappings. Then $A, B, S$ and $T$ have a unique common fixed point.

Proof: Let $x_{0}$ be any fixed point in $X$. Define sequences $\left\{x_{n}\right\}$ and $\left\{y_{n}\right\}$ in $X$ given by the rule $y_{2 n}=A x_{2 n}=T x_{2 n+1}$ and $y_{2 n+1}=B x_{2 n+1}=S x_{2 n+2}$.

As in theorem 3.1, the sequence $\left\{y_{n}\right\}$ is a Cauchy sequence in $X$. Since $X$ is complete, there is a point $z$ in $X$ such that

$$
y_{2 n}=A x_{2 n}=T x_{2 n+1} \rightarrow z \text { and } y_{2 n+1}=B x_{2 n+1}=S x_{2 n+2} \rightarrow z \text { as } n \rightarrow \infty .
$$

Now, suppose that $A$ and $S$ are $\psi$-compatible pair of reciprocal continuous mappings, so we have $A S x_{2 n} \rightarrow A z$ and $S A x_{2 n} \rightarrow S z$

Also, $\psi$-compatibility of $A$ and $S$ implies that

(3.12) $\lim _{n \rightarrow \infty} \psi\left(M\left(A S x_{2 n}, S A x_{2 n}, t\right)\right)=1$

By lemma (2.3) $M\left(S A x_{2 n}, A S x_{2 n}, t\right) \rightarrow 1$ as $n \rightarrow \infty$. We claim that $S z=A z$.

(3.13) $M(S z, A z, t)=\lim _{n \rightarrow \infty} M\left(S A x_{2 n}, A S x_{2 n}, t\right) \rightarrow 1 \Rightarrow S z=A z$.

Since, $A X \subset T X$, there is a point $w$ in $X$ such that $T w=A z$. By (3.13),

(3.14) $T w=A z=S z$

Now, we show that $B w=A z$. Suppose $B w \neq A z$. Using (3.11), we have

$$
\begin{aligned}
& \psi(M(A z, B w, h t)) \leq M_{\psi}(z, w, t) \\
& =\psi(M(B w, T w, t))=\psi(M(B w, A z, t))
\end{aligned}
$$

A contradiction. Hence $B w=A z$. Therefore by (3.14)

(3.15) $T w=A z=S z=B w$.

As $A$ and $S$ are weakly commuting, we have $A S z=S A z$ and hence

(3.16) $A A w=A S w=S A w=S S w$.

Also, $B$ and $T$ are weakly commuting, we get

(3.17) $B B w=B T w=T B w=T T w$.

Finally, we show that $A A z=A z$. Again using (3.11) with $\alpha=1$.

$$
\begin{aligned}
& \psi(M(A A z, A z, h t))=\psi(M(A A z, B w, h t)) \\
& \leq M_{\psi}(A z, w, t)=\psi(M(A A z, B w, t))
\end{aligned}
$$

which gives that $A A z=A z$. Therefore, $A z$ is a common fixed point of $A$ and $S$. Similarly, we can show that $B B w=B w$ and since $T B w=B B w$, we have $B w$, a common fixed point of $B$ and $T$. Finally, $A z=B w$, we have $A z$ as a common fixed point for $A, B, S$ and $T$. The uniqueness follows from 2) and hence the theorem.

\section{Conflict of Interest}

All the authors declare that they have no conflict of interest.

\section{Acknowledgements}

The authors wish to acknowledge with thanks the Deanship of Scientific Research, Jazan University, Jazan, K.S.A., for their technical and financial support for this research.

\section{References}

[1] Kramosil, I. and Michalek, J. (1975) Fuzzy Metric and Statistical Metric Spaces. Kybernetika, 11, 326-334. 
[2] Grabiec, M. (1988) Fixed Points in Fuzzy Metric Spaces. Fuzzy Sets and Systems, 27, 385-389. http://dx.doi.org/10.1016/0165-0114(88)90064-4

[3] George, A. and Veeramani, P. (1994) On Some Results in Fuzzy Metric Space. Fuzzy Sets and Systems, 64, $395-399$. http://dx.doi.org/10.1016/0165-0114(94)90162-7

[4] Boyd, W. and Wong, S.W. (1969) On Nonlinear Contractions. Proceedings of the American Mathematical Society, 20, 458-464. http://dx.doi.org/10.1090/S0002-9939-1969-0239559-9

[5] Alber, Y.I. and Guerre-Delabriere, S. (1997) Principle of Weakly Contractive Maps in Hilbert Spaces. In: Gohberg, I. and Lyubich, Y., Eds., New Results in Operator Theory and Its Applications, Vol. 98 of Operator Theory, Advances and Applications, Birkhauser, Basel, 7-22.

[6] Khan, M.S., Swaleh, M. and Sessa, S. (1984) Fixed Point Theorems by Altering Distances between the Points. Bulletin of the Australian Mathematical Society, 30, 1-9. http://dx.doi.org/10.1017/S0004972700001659

[7] Pant, R.P., Jha, K. and Lohani, A.B. (2003) A Note on Common Fixed Points by Altering Distances. Tamkang Journal of Mathematics, 34, 59-62.

[8] Pant, R.P., Jha, K. and Pande, V.P. (2003) Common Fixed Point for by Altering Distances between Points. Bulletin of Calcutta Mathematical Society, 95, 421-428.

[9] Pant, R.P., Jha, K. and Padaliya, S. (2003) On Common Fixed Point by Altering Distances between the Points. Tamkang Journal of Mathematics, 34, 239-243.

[10] Sumitra, Chauhan, S. and Kadelburg, Z. (2013) A Common Fixed Point Theorem in Metric Space under General Contractive Condition. Journal of Applied Mathematics, 2013, Article ID: 510691, 7 p. http://dx.doi.org/10.1155/2013/510691

[11] Sumitra, Imdad, M. and Chauhan, S. (2013) Unified Fixed Point Theorems via Common Limit Range Property in Modified Intuitionistic Fuzzy Metric Spaces. Hindawi Publishing Corporation, Abstract and Applied Analysis, 2013, Article ID: 413473, 11 p.

[12] Sumitra, Manro, S., Bhatia, S.S., Kumar, S. and Kumum, P. (2013) Weakly Compatibly Mapping with CLRS Mapping in Fuzzy-Metric Spaces. Journal Nonlinear Analysis and Applications, 2013, 1-12.

[13] Sastry, K.P.R., Naidu, S.V.R., Babu, G.V.R. and Naidu, G.A. (2000) Generalization of Common Fixed Point Theorems for Weakly Commuting Maps by Altering Distances. Tamkang Journal of Mathematics, 31, 243-250.

[14] Vasuki, R. (1999) Common Fixed Points for R-Weakly Commuting Mappings in Fuzzy Metric Spaces. Indian Journal of Pure and Applied Mathematics, 30, 419-423.

[15] Abbas, M., Imdad, M. and Gopal, D. (2011) $\psi$-Weak Contractions in Fuzzy Metric Spaces. Iranian Journal of Fuzzy Systems, 8, 141-148.

[16] Vetro, C. and Vetro, P. (2008) Common Fixed Points for Discontinuous Mappings in Fuzzy Metric Spaces. Rendiconti del Circolo Matematico di Palermo, 57, 295-303.

[17] Jha, K., Abbas, M., Beg, I., Pant, R.P. and Imdad, M. (2011) Common Fixed Point Theorem for ( $\phi$, $\psi$ )-Weak Contractions in Fuzzy Metric Spaces. Bulletin of Mathematical Analysis and Applications, 3, 149-158.

[18] Sharma, P. and Chandel, R.S. (2013) Reciprocally Continuous Maps in a Fuzzy Metric Space Involving Implicit Relations. Journal of Advanced Studies in Topology, 4, 32-39. 